\title{
Perfiles de aprendizaje musical formal e informal en educación superior ${ }^{1}$
}

\author{
Rubén Carrillo²; Patricia A. González-Moreno ${ }^{3}$
}

Recibido: 20 de febrero de 2020 / Aceptado: 28 de julio de 2020

Resumen. El propósito del presente estudio fue examinar los perfiles motivacionales de estudiantes universitarios con relación al uso de estrategias de aprendizaje musical formal e informal, así como analizar sus trayectorias de formación y el cómo estas influyen en sus preferencias y las actividades musicales que realizan dentro y fuera de la escuela. En esta investigación participaron 132 estudiantes de programas de licenciatura en música provenientes de dos universidades mexicanas. Los participantes fueron clasificados en tres grupos de acuerdo con sus experiencias musicales y su formación previa: formal, informal y mixto. Se adaptó una escala psicométrica basada en la Teoría de Expectativas y Valores de Eccles et al., para examinar la motivación estudiantil hacia el uso de estrategias de aprendizaje formal (lectura musical, interpretación de repertorio, tocar y practicar solo) e informal (tocar de oído, improvisación, tocar en grupo). Los perfiles motivacionales obtenidos sugieren diferencias significativas entre grupos en su percepción hacia diversas estrategias de aprendizaje, de las cuales tocar de oído, improvisar y tocar en grupo tienden a ser favorecidas por los grupos informal y mixto, mientras que el grupo formal favorece más estrategias como la lectura musical o la interpretación fiel a la partitura. La percepción de dificultad de estas tareas también difiere entre los grupos. Estos resultados apuntan a la necesidad de dimensionar una mayor inclusión de estrategias de aprendizaje y géneros musicales dentro de la educación formal en nivel superior que atiendan a las necesidades e intereses del alumnado.

Palabras clave: Aprendizaje musical informal; música popular; tocar de oído; improvisación musical; estrategias de aprendizaje musical.

\section{[en] Formal and informal music learning profiles in higher education}

Abstract. The aim of this study was to examine the motivational profiles of university students in relation to the use of formal and informal musical learning strategies, and to analyze their training trajectories and how they influenced their preferences and musical activities they perform inside and outside of school. A total of 132 undergraduate music students from two Mexican universities participated in this study. Participants were classified into three groups according to their previous musical experiences and training: formal, informal, and mixed. Based on Eccles et al.'s Expectancy-Value Theory, a scale was adapted to examine students' motivation to use formal (music reading, strict score-based interpretation, solo practice by playing alone) and informal learning strategies (playing by ear, improvisation, playing with others). Their motivational profiles suggest statistically significant differences among groups in their perception towards various learning strategies: playing by ear, improvising, and playing in groups were favored by informal and mixed groups, while the formal group favored strategies such as music reading and score-based performance. Perceptions of difficulty about these tasks were also different among groups. These results suggest the need of including a wider range of learning strategies and musical genres within formal higher education that meet students' needs and interests.

Keywords: Informal music learning; popular music; play by ear; music improvisation; music learning strategies.

Sumario. 1. Introducción 2. El modelo de expectativas y valores y su aplicación en la educación musical. 3. Método. 4. Resultados. 5. Discusión. 6. Conclusiones. 7. Referencias bibliográficas.

Esta investigación fue financiada por el Programa Nacional de Posgrados de Calidad (PNPC) del Consejo Nacional de Ciencia y Tecnología (CONACYT), México, a través del Doctorado en Educación, Artes y Humanidades de la Universidad Autónoma de Chihuahua, México.

2 Universidad Autónoma de Chihuahua (México)

E-mail: p215355@uach.mx

https://orcid.org/0000-0002-4105-8079

3 Universidad Autónoma de Chihuahua (México)

E-mail: pagonzalez@uach.mx

https://orcid.org/0000-0003-4529-0091

Rev. electrón. complut. inves. educ. music. 18, 2021: 139-152 
Cómo citar: Carrillo, R., y González Moreno, P. A. (2021). Perfiles de aprendizaje musical formal e informal en educación superior. Revista Electrónica Complutense de Investigación en Educación Musical, 18, 139-152. http://dx.doi.org/10.5209/ reciem. 67923

\section{Introducción}

Los programas de formación musical a nivel profesional en México, al igual que en otros países latinoamericanos como Chile, Argentina y Colombia, se encuentran fuertemente influenciados por la tradición eurocentrista y el modelo de enseñanza de conservatorio (Alessandroni, 2013; Bermúdez y Duque, 2000; Burcet, 2017; González-Moreno, 2015; Green, 2002; Holguín-Tovar y Martínez, 2017; Poblete Lagos, 2019; Shifres y Gonnet, 2015; Shifres y Holguín-Tovar, 2015), el cual se enfoca en la enseñanza de habilidades de ejecución vocal o instrumental y en la formalización de la alfabetización musical mediante la enseñanza de la notación convencional occidental. Este modelo privilegia la enseñanza de la música clásica y ciertas prácticas asociadas a ella, tales como un enfoque predominante en la lectura (Burcet, 2017; Lehmann, Sloboda, y Woody, 2007; Puurtinen, 2018; Shifres, 2018; Woody, 2007, 2012), el uso de repertorio de compositores clásicos (Burnard, 2012; Sarath, Myers y Campbell, 2016; Woody, 2007), y en la mera interpretación de dicho material (Sarath et al., 2016).

En contraste, dentro del campo de la música popular y la educación musical informal, los músicos se han valido de una amplia variedad de estrategias para desarrollar las habilidades necesarias para su adecuado desempeño profesional (Green, 2002), tales como el aprendizaje mediante la interacción con otros músicos, el uso de métodos de aprendizaje autodidacta, además de procesos de enculturamiento (Green, 2002; Lehmann et al., 2007; Vitale, 2011). Los ambientes informales de aprendizaje se caracterizan además por favorecer el aprendizaje de oído y la imitación (Green, 2002; Woody, 2007), el trabajo colaborativo y desarrollo de la creatividad (Burnard, 2012; Green, 2002; Woody, 2007). Debido a que los géneros musicales populares (con excepción del jazz) no han tenido el reconocimiento dentro de los programas curriculares de las instituciones educativas, los músicos los han aprendido de manera informal.

Por tales diferencias entre los estilos de enseñanza y aprendizaje en ambientes formales e informales, así como las actitudes y valores hacia cada una de estas tradiciones, se ha dado una separación entre ambos tipos de músicos: los académicos y los populares, a pesar de que los recursos, métodos y estrategias de aprendizaje informales pudieran ser aprovechados dentro de los contextos formales (Carrillo y González-Moreno, 2019; Green, 2002; Heckel, 2017; Hewitt, 2018; Virkkula, 2015; Virkkula y Kunwar, 2016; Wright, 2016). Por esta razón, en esta investigación se considera pertinente analizar los efectos que tiene la motivación de estudiantes de música en nivel licenciatura, en relación con el uso de estrategias de aprendizaje formal e informal, y cómo esto afecta el desarrollo de sus aptitudes musicales en el contexto educativo formal.

La presente investigación busca responder a las siguientes preguntas: (a) ¿Cuáles son las experiencias musicales previas que se relacionan en mayor medida con la utilización de estrategias formales e informales de aprendizaje musical? (b) ¿Qué diferencias existen en las percepciones de valor y expectativas de éxito hacia diversas estrategias de aprendizaje musical, por parte del alumnado, en función de su tipo de formación musical (formal, informal y mixto)? y (c) ¿En qué medida se relaciona la motivación hacia las estrategias de aprendizaje musical formales e informales con el desarrollo de aptitudes musicales de estudiantes de licenciatura dentro de contextos formales?

\section{El modelo de expectativas y valores y su aplicación en la educación musical}

El marco teórico que sustenta esta investigación es el Modelo de Expectativas y Valores inicialmente denominado Eccles' Expectancy Value Model of Achievement Choices (Eccles et al., 1983; Eccles et al., 1993; Wigfield y Eccles, 2000, 2020), y que posterior a la realización de este estudio ha sido renombrado como Situated Expectancy-Value Theory, SEVT (Eccles y Wigfield, 2020). Este modelo ha servido de referente en otras investigaciones dentro del campo de la educación musical (González-Moreno, 2010, 2012; McPherson y Hendricks, 2010; McPherson y O’Nei11 2010; McPherson, Osborne, Barrett, Davidson y Faulkner, 2015; Mohd y McPherson, 2009; O’Neill y McPherson, 2002; Parkes y Jones, 2012; Uy, 2018).

Con este marco teórico se ha realizado diversas investigaciones, tales como examinar las actitudes de alumnos en nivel básico y medio superior en relación con el valor que le atribuyen a estudiar música en comparación con otras materias escolares (ver McPherson y O’Neill, 2010), ejecución musical (McCormick y McPherson, 2007; O’Neill y McPherson, 2002), o el rol de los padres en la educación musical de los niños (McPherson, 2009) por mencionar algunos ejemplos. Sin embargo, no se encontró bibliografía donde se haya utilizado dicha teoría para medir las actitudes y valores en cuanto a estrategias de aprendizaje musical formales e informales.

\subsection{Expectativas de éxito}

Las expectativas de éxito se definen como la autopercepción del individuo acerca de su capacidad para desarrollar una tarea exitosamente (Eccles et al., 1983; Wigfield y Eccles, 2000, 2020; O’Neill y McPherson, 2002), lo que infiere que 
a mayores expectativas mayor el esfuerzo y persistencia invertidos para el logro de la tarea. Las expectativas positivas favorecen la motivación a realizar dicha actividad mientras que las negativas tienden a desalentarla (Uy, 2018).

De acuerdo con Eccles y Wigfield (2020), estas expectativas surgen a partir del autoconcepto sobre las propias habilidades, autoesquemas de las identidades personales y sociales, e incluso en función de las metas a corto y largo plazo. Por lo tanto, este constructo está directamente relacionado tanto con el sentido de competencia o auto-eficacia (Bandura, 2005), expresado como la autopercepción acerca de las habilidades propias, la cual puede afectar la elección de las actividades a desarrollar (Eccles et al., 1983; Wigfield y Eccles, 2000; Wigfield y Eccles, 2020), como con la percepción del individuo sobre la dificultad de la tarea (Eccles et al., 1983; Eccles y Wigfield, 2020), la cual está inversamente relacionada con el sentido de competencia. Por ejemplo, aquellos sujetos que tienen una baja apreciación de sus habilidades para desempeñarse adecuadamente en cierta actividad también tienden a percibir dicha tarea como de mayor dificultad (Eccles et al., 1983; González-Moreno, 2010; McPherson y O'Neill, 2010; McPherson et al., 2015). Estos sub-constructos en su conjunto son considerados predictores del desempeño en dicha tarea (Eccles y Wigfield, 2020).

\subsection{Valores subjetivos atribuidos a la tarea}

Adicionalmente a las expectativas de éxito, la motivación para realizar una tarea se encuentra influenciada por las percepciones de valor que el individuo tenga sobre la misma (Eccles et al., 1983, Eccles y Wigfield, 2020). Este constructo está compuesto por cuatro elementos principales: (a) interés, o valor intrínseco de la tarea, relacionado con el disfrute experimentado al realizar la actividad; (b) importancia o valor de logro, definido como el valor atribuido a realizar bien una tarea (Eccles et al., 1983; Eccles y Wigfield, 2002, 2020); esto es, "si el estudiante cree que es importante desempeñarse bien en dicha tarea, entonces esta tiene un alto valor de logro..." (Uy, 2018, p. 32); y (c) la utilidad, relacionada con el valor utilitario que se puede obtener de realizar dicha tarea (Eccles et al., 1983; Eccles y Wigfield, 2002, 2020); y (d) el costo atribuido a participar en la tarea, tanto en relación al esfuerzo, al costo emocional o al costo de dejar una actividad por otra (Eccles y Wigfield, 2020).

Dentro de este estudio cuantitativo, descriptivo y exploratorio, se examinaron los constructos de expectativas de éxito (expectativas, junto con sentido de competencia y dificultad de la tarea) y valor subjetivo de la tarea (interés, importancia y utilidad) de dicha teoría. Debido a que el costo de participar en una tarea es un subconstructo multidimensional complejo (Eccles y Wigfield, 2020), y que no se buscaba profundizar en las interrelaciones de este con los otros subconstructos, se decidió omitir su inclusión en el instrumento de investigación. Además, aunque la teoría contempla un modelo muy complejo desde el contexto sociocultural, pasando por los procesos cognitivos (percepciones sobre el contexto e interpretaciones de experiencias pasadas) y la generación de las creencias motivacionales del individuo, este estudio se focalizó exclusivamente en los constructos anteriormente descritos, como predictores de las decisiones que el individuo toma para la consecución de sus metas.

\section{Método}

En esta investigación participaron 132 estudiantes de licenciatura en música provenientes de dos universidades ubicadas, una en el norte y otra en el centro de México. El estudio fue realizado con estudiantes de segundo y cuarto semestre, participando 77 hombres y 55 mujeres (58.3\% y 41.7\%, respectivamente). El rango de edad del grupo participante era de 18 a 47 años, en tanto que el $75 \%$ de ellos tenían entre 18 y 22 años.

Con base en la información obtenida a través del instrumento de investigación, los participantes fueron clasificados en tres grupos de acuerdo con sus experiencias musicales y formación previa, clasificación utilizada para analizar estadísticamente sus perfiles motivacionales: (1) estudiantes con formación principalmente clásica, sin experiencia en música popular (denominado como "Formal"; $n=44,33.33 \%$ ); (2) estudiantes con entrenamiento formal pero con experiencia en música popular mayor a tres años ("Informal"; $n=37,28.03 \%$ ), (c) estudiantes con estudios formales pero con experiencia en música popular limitada, menor a tres años ("Mixto"; $n=51,38.63 \%$ ).

\subsection{Instrumento}

$\mathrm{Al}$ alumnado le fue aplicada una escala psicométrica basada en la Teoría de Expectativas y Valores de Eccles et al. (1983, 1993), la cual fue inicialmente adaptada por McPherson y colegas (McPherson y O'Neill, 2010; McPherson et al., 2015) para investigar la motivación hacia el estudio de la música, y que en este estudio se enfocó en la motivación hacia el empleo de estrategias formales e informales de aprendizaje musical. En la primera sección se buscó recoger datos generales acerca de la formación musical del alumnado y sus experiencias previas, mientras que la segunda sección buscó medir los perfiles motivacionales hacia seis estrategias de aprendizaje musical, tres de ellas fuertemente relacionadas con el aprendizaje musical formal: (1) la lectura ensayada (Lehmann et al., 2007; Woody, 2007, 2012), (2) interpretación musical apegada a cómo la música fue escrita (Sarath et al., 2016) y (3) tocar solo (estudiar de manera individual); y las tres estrategias restantes relacionadas con el aprendizaje informal: (4) tocar de oído (Green, 2002; Woody, 2007), (5) improvisación y (6) tocar en grupo (Burnard, 2012; Green, 2002; Woody, 2007). 
El instrumento utilizado fue previamente analizado en una prueba piloto para determinar sus niveles de validez y confiabilidad. El proceso de validación del instrumento se describe en mayor detalle en Carrillo y González-Moreno (2019). La validez del instrumento fue evaluada de cuatro formas distintas: (a) validez de constructo; (b) validez de contenido; (c) validez de aspecto; y (d) validez discriminante mediante análisis factorial, donde los valores KMO obtenidos se encontraron en el rango de 0.752 y 0.904 para la prueba piloto, considerándose valores superiores a 0.7 como adecuados (Beavers et al., 2013). De manera similar a lo realizado en el estudio piloto, se realizó la reducción de variables mediante análisis factorial, los índices de KMO y de esfericidad de Bartlett permanecieron dentro de rangos muy buenos a sobresalientes, e incluso superiores a los obtenidos en la prueba piloto (Tabla 1).

Tabla 1. Índices de Kaiser-Meyer-Olkin y Esfericidad de Bartlett para cada una de las estrategias de aprendizaje.

\begin{tabular}{|c|c|c|c|c|c|c|}
\hline Parámetro & $\begin{array}{c}\text { Tocar de } \\
\text { Oído }\end{array}$ & $\begin{array}{c}\text { Improvisa- } \\
\text { ción }\end{array}$ & $\begin{array}{c}\text { Tocar en } \\
\text { grupo }\end{array}$ & Lectura & $\begin{array}{c}\text { Interpreta- } \\
\text { ción }\end{array}$ & Tocar solo \\
\hline Índice KMO & 0.9 & 0.9 & 0.89 & 0.91 & 0.79 & 0.8 \\
\hline $\begin{array}{c}\text { Esfericidad de } \\
\text { Barlett }\end{array}$ & 0 & 0 & 0 & 0 & 0 & 0 \\
\hline
\end{tabular}

En el estudio final, los índices de confiabilidad interna de la escala para los diversos constructos, medidos a través del $\alpha$ de Cronbach, fueron favorables y se encontraron en un rango de .694 a .911.

\section{Resultados}

\subsection{Experiencias musicales en su formación y práctica profesional}

Los participantes fueron cuestionados acerca de si trabajaban fuera de la escuela en alguna actividad relacionada con la música. Mediante una prueba de bondad de ajuste de Chi-cuadrada $\left(\chi^{2}\right)$ se encontraron diferencias significativas en sus respuestas $(p=.024$; a un nivel de alfa $=.05)$. Los resultados sugieren que la gran mayoría del alumnado con formación exclusivamente formal no trabajaban en actividades musicales ( $n=31,70.5 \%$ del grupo formal), mientras que más de la mitad de discentes de los grupos informal y mixto sí laboraban en actividades musicales $(n=19$, $52.8 \% ; n=28,56.0 \%$, respectivamente).

Además de las actividades laborales, se preguntó al alumnado acerca de su participación en agrupaciones musicales (clásicos o populares) fuera de la escuela, donde las diferencias de distribución entre los grupos fueron estadísticamente significativas $(p<.01)$. Los resultados obtenidos muestran que un porcentaje muy superior de estudiantes de los grupos informal y mixto habían tenido experiencia musical previa en agrupaciones musicales fuera de la escuela ( $n=31,86.1 \%$, y $n=47,92.2 \%$, respectivamente), en comparación con un porcentaje menor de estudiantes del grupo formal que reportaban haber tenido dicha experiencia $(n=24,57.1 \%)$.

Con relación al consumo musical, se encontraron diferencias significativas entre los grupos en géneros como música clásica (periodos barroco, clásico, romántico e impresionista) $(p<.01)$, música clásica contemporánea (segunda mitad del siglo XX y siglo XXI) $(p<.01)$, jazz $(p<.05)$ y otros géneros musicales (música cristiana, flamenco, cinematográfica) $(p<.05)$. Los resultados obtenidos mostraron que los estudiantes del grupo formal escuchaban música clásica y contemporánea en mayor medida. El 75\% $(n=33)$ de este grupo reportaba escuchar música clásica a diario o casi a diario, a diferencia de los grupos informal y mixto en los cuales la mayoría reportaron que la escuchaban entre una y cuatro veces por semana.

En el caso de la música clásica contemporánea, los grupos formal y mixto reportó escucharla más, a diferencia del grupo informal, donde el 42.9\% $(n=15)$ reportaron no escucharla nunca. En el caso del jazz, el grupo informal reportó una mayor escucha con más del 67\% $(n=33)$. Finalmente, en el caso de otros géneros no mencionados en la encuesta, dentro de los cuales los participantes hicieron mención a géneros como música cristiana, música de filmes y k-pop, se encontró un mayor consumo entre los grupos informal y mixto, en ambos casos con 60 a $66 \%$ de ellos (n $=9$, y $n=8$, respectivamente), refiriendo a que escuchaban este tipo de géneros a diario o casi diario, mientras que el grupo formal, más del $83 \%(n=15)$ refirió que no escuchaban ningún tipo de género adicional no mencionado en el instrumento.

Del mismo modo, se cuestionó a los participantes acerca de su experiencia como músicos ejecutantes en diversos géneros musicales. Después de una prueba de Chi cuadrada $\left(\chi^{2}\right)$ se encontraron diferencias estadísticamente significativas entre los grupos en géneros como la música clásica, rock, metal y banda sinaloense $(p<.01)$. Los participantes del grupo formal reportaron tener una mayor experiencia en el género de música clásica con un 77\% $(n=34)$, en contraste al $24.3 \%(n=9)$ y $27.5 \%(n=14)$ de los grupos informal y mixto, respectivamente. El caso contrario ocurre en la música pop, donde el 65.9\% $(n=29)$ de participantes del grupo formal reportó no tener experiencia, en contraste con los grupos informal y mixto cuyos integrantes reportaron contar con experiencia en ese género con $62 \%(n=23)$ 
y $67 \%(n=34)$, respectivamente. Lo mismo sucede con otros géneros de música popular como el rock, metal y banda sinaloense, donde la mayoría de integrantes de los grupos informal y mixto reportaron tener experiencia a diferencia de los músicos del grupo formal.

En cuanto a las actividades realizadas dentro de la escuela, se encontraron algunas diferencias estadísticamente significativas $(p<.01)$ en cuanto a la frecuencia de realización de actividades tales como improvisar, tocar en grupo y tocar de oído. En la Tabla 2, se observa como el 41.9\% $(n=18)$ de integrantes del grupo formal reportaron que nunca realizaban actividades de improvisación y el 39.5\% $(n=17)$ reportaron hacerlo sólo 1 o 2 veces por semana. Por el contrario, en el caso del grupo informal, el 32.4\% de integrantes $(n=12)$ reportó hacerlo a diario mientras otro $32.4 \%(n=12)$ dijo hacerlo por lo menos 3 o 4 veces por semana. En el caso de tocar en grupo o ensamble, el $25.6 \%$ $(n=11)$ de integrantes del grupo formal reportaron nunca utilizar esta estrategia de aprendizaje; estos porcentajes son menores en los grupos informal y mixto, con un $13.5 \%(n=5)$ y $9.8 \%(n=5)$ respectivamente. En el caso de tocar de oído, sólo un $9.1 \%(n=4)$ de los integrantes del grupo formal reportó hacerlo a diario, a diferencia de un $34.3 \%$ $(n=12)$ del grupo informal.

Tabla 2. Frecuencia en el uso de estrategias de aprendizaje musical dentro de la escuela.

\begin{tabular}{|c|c|c|c|c|c|c|c|c|c|c|}
\hline \multirow{2}{*}{ Actividad } & \multicolumn{2}{|c|}{ Nunca } & \multicolumn{2}{|c|}{$1-2$ veces } & \multicolumn{2}{|c|}{$3-4$ veces } & \multicolumn{2}{|c|}{$5-6$ veces } & \multicolumn{2}{|c|}{ Diario } \\
\hline & $f$ & $\%$ & $f$ & $\%$ & $f$ & $\%$ & $f$ & $\%$ & $f$ & $\%$ \\
\hline \multicolumn{11}{|c|}{ Improvisación** } \\
\hline Formal & 18 & 41.9 & 17 & 39.5 & 3 & 7.0 & 0 & 0 & 5 & 11.6 \\
\hline Informal & 5 & 13.5 & 3 & 8.1 & 12 & 32.4 & 5 & 13.5 & 12 & 32.4 \\
\hline Mixto & 11 & 21.6 & 20 & 39.2 & 8 & 15.7 & 5 & 9.8 & 7 & 13.7 \\
\hline \multicolumn{11}{|l|}{ Lectura } \\
\hline Formal & 0 & 0 & 2 & 4.5 & 3 & 6.8 & 7 & 15.9 & 32 & 72.7 \\
\hline Informal & 0 & 0 & 5 & 13.5 & 8 & 21.6 & 10 & 27.0 & 14 & 37.8 \\
\hline Mixto & 1 & 2.0 & 5 & 9.8 & 4 & 7.8 & 14 & 27.5 & 27 & 52.9 \\
\hline \multicolumn{11}{|l|}{ Ensamble** } \\
\hline Formal & 11 & 25.6 & 9 & 20.9 & 17 & 39.5 & 4 & 9.3 & 2 & 4.7 \\
\hline Informal & 5 & 13.5 & 5 & 13.5 & 14 & 37.8 & 8 & 21.6 & 5 & 13.5 \\
\hline Mixto & 5 & 9.8 & 26 & 51.0 & 9 & 17.6 & 9 & 17.6 & 2 & 3.9 \\
\hline \multicolumn{11}{|l|}{ Interpretación } \\
\hline Formal & 3 & 6.8 & 6 & 13.6 & 8 & 18.2 & 14 & 31.8 & 13 & 29.5 \\
\hline Informal & 3 & 8.1 & 12 & 32.4 & 8 & 21.6 & 7 & 18.9 & 7 & 18.9 \\
\hline Mixto & 5 & 9.8 & 10 & 19.6 & 11 & 21.6 & 12 & 23.5 & 13 & 25.5 \\
\hline \multicolumn{11}{|c|}{ Tocar de oído** } \\
\hline Formal & 9 & 20.5 & 17 & 38.6 & 9 & 20.5 & 5 & 11.4 & 4 & 9.1 \\
\hline Informal & 5 & 14.3 & 7 & 20.0 & 5 & 14.3 & 6 & 17.1 & 12 & 34.3 \\
\hline Mixto & 7 & 13.7 & 7 & 13.7 & 23 & 45.1 & 4 & 7.8 & 10 & 19.6 \\
\hline \multicolumn{11}{|l|}{ Tocar solo } \\
\hline Formal & 1 & 2.3 & 6 & 13.6 & 9 & 20.5 & 8 & 18.2 & 20 & 45.5 \\
\hline Informal & 3 & 8.1 & 4 & 10.8 & 6 & 16.2 & 11 & 29.7 & 13 & 35.1 \\
\hline Mixto & 3 & 5.9 & 3 & 5.9 & 9 & 17.6 & 12 & 23.5 & 24 & 47.1 \\
\hline
\end{tabular}

Nota. $* *$ significancia $p<.01$.

De manera similar, el cuerpo estudiantil fue cuestionado acerca de la frecuencia en el uso de las estrategias de aprendizaje musical investigadas en este estudio fuera del contexto escolar (Tabla 3). Para la actividad de improvisación, nuevamente los resultados sugieren que fuera de la escuela era una práctica más común para los integrantes del grupo informal donde el 40.5\% $(n=15)$ reportó hacerlo a diario, a diferencia del 9.3\% $(n=4)$ reportado por los integrantes del grupo formal $(p<.01)$.

Lo mismo se observó con la actividad de tocar en grupo (ensamble), donde sólo un 2.3\% $(n=1)$ de los integrantes del grupo formal reportó tocar a diario en agrupaciones musicales fuera de la escuela, contra un $22.2 \%(n=8)$ del grupo informal. Además, un 60.5\% $(n=26)$ de integrantes del grupo formal reportaron no tocar en grupo fuera de la escuela, este porcentaje es significativamente mayor en comparación con el grupo informal, con un 19.4\% $(n=7)$. 
Tabla 3. Frecuencia en el uso de estrategias de aprendizaje musical fuera de la escuela.

\begin{tabular}{|c|c|c|c|c|c|c|c|c|c|c|}
\hline \multirow{2}{*}{ Actividad } & \multicolumn{2}{|c|}{ Nunca } & \multicolumn{2}{|c|}{$1-2$ veces } & \multicolumn{2}{|c|}{$3-4$ veces } & \multicolumn{2}{|c|}{$5-6$ veces } & \multicolumn{2}{|c|}{ Diario } \\
\hline & $f$ & $\%$ & $f$ & $\%$ & $f$ & $\%$ & $f$ & $\%$ & $f$ & $\%$ \\
\hline \multicolumn{11}{|l|}{ Lectura } \\
\hline Formal & 0 & 0 & 7 & 16.3 & 5 & 11.6 & 13 & 30.2 & 18 & 41.9 \\
\hline Informal & 2 & 5.4 & 10 & 27.0 & 10 & 27.0 & 3 & 8.1 & 12 & 32.4 \\
\hline Mixto & 2 & 3.9 & 7 & 13.7 & 16 & 31.4 & 7 & 13.7 & 19 & 37.3 \\
\hline \multicolumn{11}{|l|}{ Ensamble** } \\
\hline Formal & 26 & 60.5 & 8 & 18.6 & 6 & 14.0 & 2 & 4.7 & 1 & 2.3 \\
\hline Informal & 7 & 19.4 & 8 & 22.2 & 7 & 19.4 & 6 & 16.7 & 8 & 22.2 \\
\hline Mixto & 15 & 30.0 & 19 & 38.0 & 11 & 22.0 & 3 & 6.0 & 2 & 4.0 \\
\hline \multicolumn{11}{|l|}{ Interpretación } \\
\hline Formal & 7 & 15.9 & 8 & 18.2 & 10 & 22.7 & 12 & 27.3 & 7 & 15.9 \\
\hline Informal & 2 & 5.6 & 11 & 30.6 & 10 & 27.8 & 6 & 16.7 & 7 & 19.4 \\
\hline Mixto & 6 & 11.8 & 4 & 7.8 & 16 & 31.4 & 16 & 31.4 & 9 & 17.6 \\
\hline \multicolumn{11}{|l|}{ Tocar de oído } \\
\hline Formal & 7 & 15.9 & 10 & 22.7 & 13 & 29.5 & 10 & 22.7 & 4 & 9.1 \\
\hline Informal & 2 & 5.4 & 7 & 18.9 & 7 & 18.9 & 7 & 18.9 & 14 & 37.8 \\
\hline Mixto & 5 & 10.0 & 8 & 16.0 & 11 & 22.0 & 12 & 24.0 & 14 & 28.0 \\
\hline \multicolumn{11}{|l|}{ Tocar solo } \\
\hline Formal & 1 & 2.3 & 6 & 13.6 & 7 & 15.9 & 10 & 22.7 & 20 & 45.5 \\
\hline Informal & 0 & 0 & 1 & 2.7 & 9 & 24.3 & 13 & 35.1 & 14 & 37.8 \\
\hline Mixto & 1 & 2.0 & 4 & 7.8 & 8 & 15.7 & 16 & 31.4 & 22 & 43.1 \\
\hline \multicolumn{11}{|c|}{ Improvisación** } \\
\hline Formal & 12 & 27.9 & 18 & 41.9 & 5 & 11.6 & 4 & 9.3 & 4 & 9.3 \\
\hline Informal & 4 & 10.8 & 2 & 5.4 & 7 & 18.9 & 9 & 24.3 & 15 & 40.5 \\
\hline Mixto & 10 & 19.6 & 12 & 23.5 & 10 & 19.6 & 8 & 15.7 & 11 & 21.6 \\
\hline
\end{tabular}

\subsection{Análisis de perfiles de los constructos motivacionales}

Se realizaron una serie de análisis multivariantes denominados Análisis de Perfiles (Profile Analysis) con el fin de examinar las diferencias entre las percepciones de valor y expectativas de éxito hacia las diversas estrategias de aprendizaje musical, en función del tipo de formación musical de los estudiantes. Este tipo de análisis es una aplicación de MANOVA en una situación donde hay más de una variable dependiente y todas ellas son medidas en la misma escala (mediciones repetidas o repeated measures) (Tabachnick y Fidell, 2007).

El método estadístico utilizado para el análisis de perfiles incluyó mediciones repetidas, el cual, aunque usualmente se utiliza para medir en pruebas de pre-post o cambios a través del tiempo, también puede ser utilizado para analizar diversas variables dependientes medidas al mismo tiempo (Tabachnick y Fidell, 2007). El análisis de perfiles busca dar respuesta a tres hipótesis: (1) determinar si existen diferencias entre los grupos con respecto a su valoración hacia las diferentes estrategias de aprendizaje; (2) determinar si existen diferencias entre las estrategias de aprendizaje; y (3) comprobar si los perfiles entre los grupos son diferentes, es decir, no existe paralelismo en sus perfiles.

Se realizó la debida revisión de los datos con el fin de comprobar que eran susceptibles de ser analizados mediante esta técnica. El análisis multivariante utilizado es robusto a violaciones de normalidad de la muestra y no presenta problemas para evaluar tamaños desiguales de muestras (Tabachnick, y Fidell, 2007). De acuerdo con Tabachnick y Fidell (2007), es necesario que el tamaño del grupo más pequeño sea mayor al número de variables dependientes a analizar, además de que es recomendable que no existan casos atípicos (outliers), aspectos que no fueron impedimento en este estudio debido a que el tamaño de la muestra fue lo suficientemente grande $(N=132)$, fueron seis las variables dependientes involucradas en los análisis y el grupo más pequeño de la muestra fue de $n$ $=37$. Adicionalmente, no se encontraron casos atípicos, esto debido al uso de escala Likert que inherentemente limita los rangos a medir. En este estudio se realizaron tres análisis de perfiles para las variables dependientes de Valor Subjetivo de la Tarea (Valores), Expectativas de Éxito (Expectativas), y Dificultad de la Tarea, que a continuación se reportan. 


\subsubsection{Valores subjetivos atribuibles a las estrategias de aprendizaje}

Se utilizó el análisis de perfiles para examinar cómo el cuerpo estudiantil valoraba las seis estrategias de aprendizaje musical contempladas en el estudio. Como variable independiente se usó el tipo de formación de los estudiantes (factor intersujetos) y como variables dependientes las valoraciones obtenidas en el cuestionario en relación con cada una de las estrategias de aprendizaje musical, tanto formal como informal (factor intrasujetos).

De acuerdo a la primera hipótesis del análisis de perfiles, que examina las diferencias de medias generales de cada grupo, no se observó significancia estadística entre ellos respecto a la valoración de las estrategias en general $[F(2$, $128)=2.17, p=.118]$, lo cual era hasta cierto punto predecible, dada la combinación de resultados, pues mientras los estudiantes con formación informal o mixto valoran más las estrategias informales, los estudiantes del grupo formal hacen lo propio con las estrategias formales, dando como resultado que las diferencias entre las medias generales sean muy pequeñas (formal, $\mathrm{M}=4.20, \mathrm{DT}=.51$; mixto, $\mathrm{M}=4.36, \mathrm{DT}=.34$; informal, $\mathrm{M}=4.37$, $\mathrm{DT}=.48$ ).

En cuanto a las diferencias sobre las valoraciones dadas a las estrategias de aprendizaje, los resultados mostraron diferencias estadísticamente significativas $[T$ de Hotelling $=.45, F(5,124)=11.14, p<.01]$, sugiriendo que las valoraciones hacia cada una de las estrategias difieren entre sí, descartando la hipótesis de similitud en la respuesta media (flatness), lo cual fácilmente se puede observar en la Figura 1, donde se muestra que no hay líneas rectas; es decir, difieren las percepciones dependiendo de la estrategia que se trate. Por ejemplo, se valora en mayor medida tocar en grupo $(\mathrm{M}=4.59, \mathrm{DT}=.05)$ que tocar solo $(\mathrm{M}=4.24, \mathrm{DT}=.07)$ o el interpretar fielmente como está escrito $(\mathrm{M}=4.03, \mathrm{DT}=.08)$.

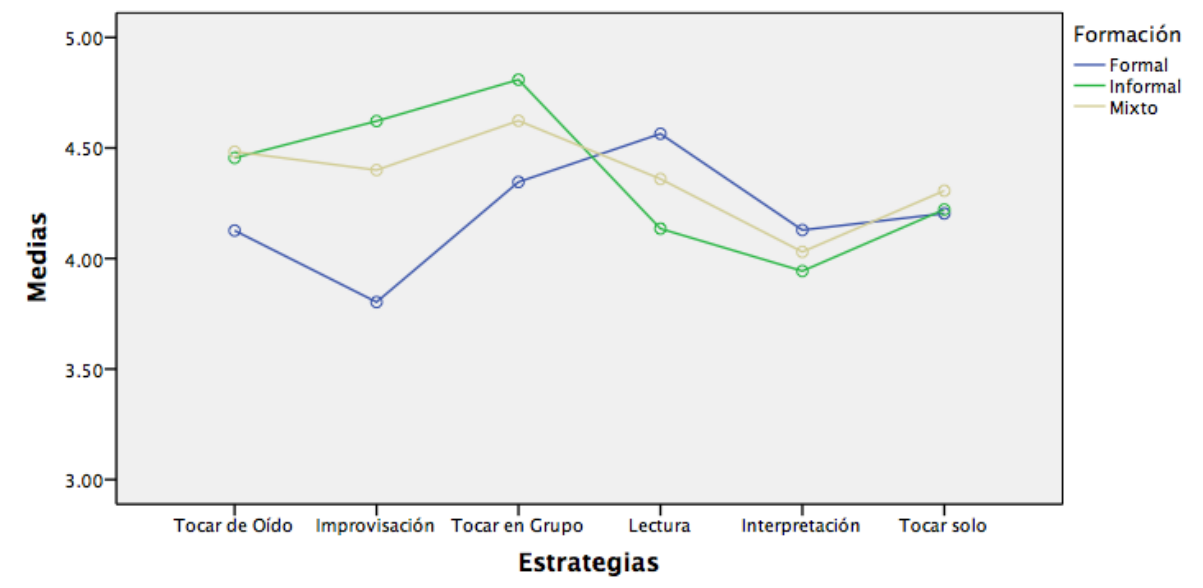

Figura 1. Percepciones de valor atribuido a estrategias de aprendizaje por tipo de formación.

Finalmente, se observan diferencias significativas entre los perfiles considerando la interacción del Tipo de Formación x Estrategia de Aprendizaje [Criterio de Pillai $=.25, F(10,250)=3.54, p<.01$ ], descartando que exista paralelismo y mostrando que existen diferencias estadísticamente significativas entre los perfiles de los grupos en función del tipo de estrategia de la que se trate. En la Figura 1 se aprecia que existe una fuerte similitud entre los grupos informal y mixto, los cuales difieren notablemente del grupo formal, lo que implica que la formación del estudiante influye en la valoración de las diversas estrategias de aprendizaje. El alumnado con una formación predominantemente formal tiende a valorar más las estrategias consideradas formales, tales como la lectura $(\mathrm{M}=4.56, \mathrm{DT}=.61)$ y la interpretación $(\mathrm{M}=4.13, \mathrm{DT}=.85)$. Sin embargo, respecto a la estrategia de tocar solo, no se observan diferencias significativas. Por su parte, estudiantes con formación informal y mixta prefieren las estrategias informales de tocar de oído (Informal, $\mathrm{M}=4.45, \mathrm{DT}=.68$; Mixto, $\mathrm{M}=4.48, \mathrm{DT}=.49$ ), improvisación (Informal, $\mathrm{M}=4.62$, DT = .65; Mixto, $\mathrm{M}=4.40, \mathrm{DT}=.68$ ) y tocar en grupo (Informal, $\mathrm{M}=4.81, \mathrm{DT}=.28 ;$ Mixto, $\mathrm{M}=4.62$, $\mathrm{DT}=.54$ ).

\subsubsection{Expectativas de éxito en relación con las estrategias de aprendizaje}

Se realizó un segundo análisis de perfiles para examinar las expectativas de éxito por parte del alumnado hacia las diversas estrategias de aprendizaje musical. Se utilizó como variable independiente el tipo de formación de los estudiantes (factor intersujetos) y como variables dependientes la valoración obtenida en el instrumento de medición con relación a cada una de las estrategias de aprendizaje musical (factor intrasujetos).

Se encontraron diferencias significativas entre los grupos respecto a las expectativas de éxito en general $[F(2$, $128)=5.3, p=.006]$, las cuales pueden observarse en la Figura 2. Las medias generales sugieren que los grupos informal y mixto $(\mathrm{M}=3.75$, $\mathrm{DT}=.63, \mathrm{y} \mathrm{M}=3.61, \mathrm{DT}=.65$, respectivamente) reportan expectativas de éxito estadísticamente más altas que el grupo formal $(\mathrm{M}=3.29$, $\mathrm{DT}=.66)$. 


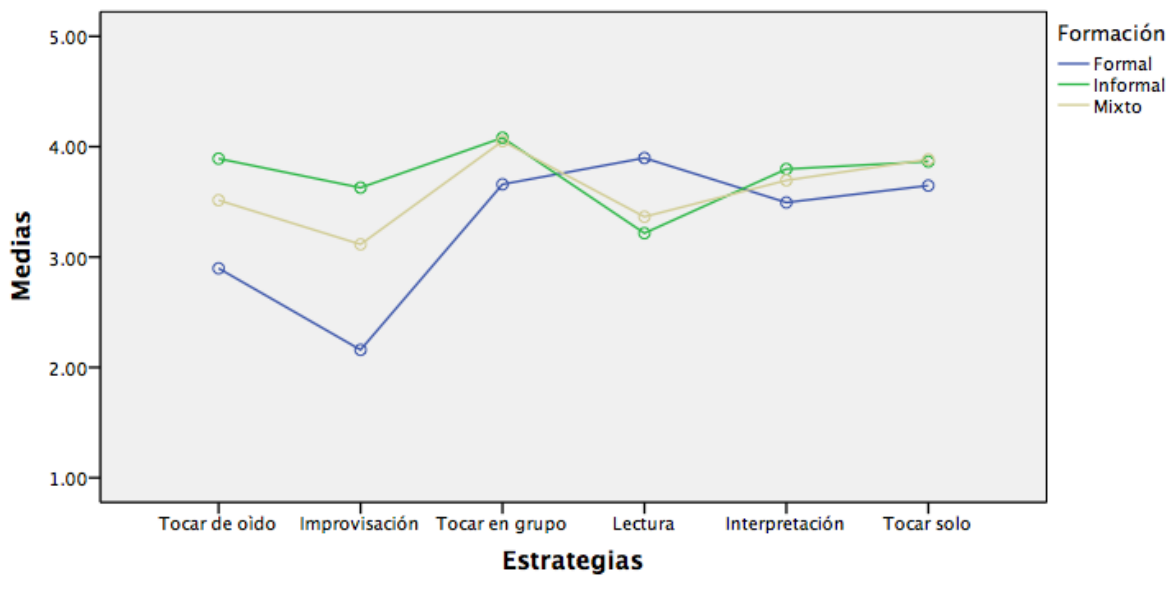

Figura 2. Percepciones de expectativas de éxito atribuido a estrategias de aprendizaje por tipo de formación.

En cuanto a las diferencias en las expectativas de éxito hacia cada una de las estrategias de aprendizaje (factor intrasujetos), los resultados también mostraron diferencias estadísticamente significativas $[T$ de Hotelling $=.74, F(5$, $124)=18.44, p<.01]$. Con esto se descarta la hipótesis de similitud en la respuesta media, ya que, como puede observarse en la Figura 2, la improvisación es la estrategia en la que el alumnado siente menos expectativas de éxito (M $=2.97$, DT $=.09$ ), en comparación con las otras estrategias de aprendizaje.

En cuanto a la prueba de paralelismo, se encontraron diferencias significativas entre los perfiles dada la interacción entre el Tipo de Formación y los Tipos de Estrategias de Aprendizaje [ $\Lambda$ Wilks $=.71, F(10,248)=4.72, p<.01]$. Al igual que en el constructo de valores, se puede observar una fuerte similitud entre los grupos informal y mixto, y un comportamiento de perfil completamente diferente para el grupo formal (ver Figura 2). Esto nos sugiere nuevamente que las expectativas de éxito hacia las diversas estrategias de aprendizaje difieren en función de la formación del estudiante. En este caso, los integrantes del grupo informal y mixto muestran, en general, un mayor sentido de competencia y una mayor confianza para desempeñarse en todas las estrategias de aprendizaje excepto en lectura (Informal, $\mathrm{M}=3.2, \mathrm{DT}=1.0$; Mixto, $\mathrm{M}=3.37$, DT = .94), donde el grupo formal reporta mayores expectativas de éxito $(\mathrm{M}=3.9, \mathrm{DT}=.87$; ver Figura 2$)$. Esto sugiere que los grupos informal y mixto tienen una mayor confianza y percepción de competencia en un rango más amplio de estrategias musicales en comparación con el grupo que solo tienen experiencia en la educación formal. En otras palabras, los grupos informal y mixto muestran una percepción más optimista de sí mismos sobre sus competencias musicales, excepto en la lectura.

\subsubsection{Percepciones sobre la dificultad de la tarea}

Se realizó un tercer análisis de perfiles en este caso para examinar las percepciones en cuanto a la dificultad de la tarea, donde, al igual que en los anteriores, se utilizó como variable independiente el tipo de formación de los estudiantes (factor intersujetos), y como variables dependientes la percepción de dificultad reportada respecto a las diversas estrategias de aprendizaje musical (factor intrasujetos).

De acuerdo a la primera hipótesis que examina las diferencias de medias generales de cada grupo, los resultados no alcanzaron significancia estadística $[F(2,127)=2.52, p=.09]$, lo que sugiere que las percepciones de dificultad hacia las estrategias en su conjunto son similares entre grupos (Formal, $\mathrm{M}=2.55, \mathrm{DT}=63$; Mixto, $\mathrm{M}=2.35$, DT = .72; Informal, $\mathrm{M}=2.21, \mathrm{DT}=.70)$. Esto puede deberse nuevamente a la combinación de resultados, debido a que quienes cuentan con formación informal o mixta perciben una menor dificultad hacia las estrategias informales, mientras que, los estudiantes del grupo formal, por su parte, perciben las estrategias informales como más difíciles. Por otra parte, analizando la Figura 3 se pueden encontrar coincidencias fuertes entre los tres grupos hacia la dificultad percibida para la estrategia de tocar en grupo y la interpretación musical.

En cuanto a las diferencias entre las medias generales de las estrategias de aprendizaje, los resultados del estudio mostraron diferencias estadísticamente significativas $[T$ de Hotelling $=.59, F(5,123)=14.54, p<.01]$, lo que sugiere que existen diferencias entre la dificultad percibida para cada una de las estrategias, descartando la hipótesis de similitud en la respuesta media. La Figura 3 muestra la ausencia de líneas rectas en los perfiles; mientras la improvisación es considerada la actividad más difícil por todos los grupos $(\mathrm{M}=2.89$, DT $=.10)$, tocar en grupo es considerada la menos difícil $(\mathrm{M}=2.00, \mathrm{SE}=.09)$. 


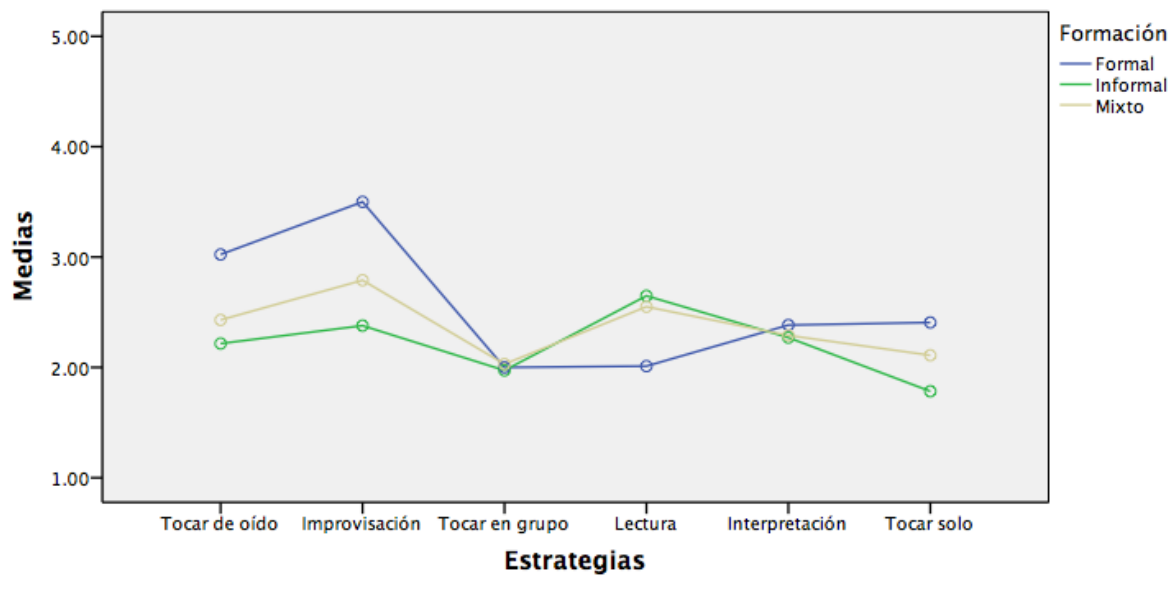

Figura 3. Percepciones de dificultad de la tarea atribuidas a cada estrategia de aprendizaje por tipo de formación musical.

Aun cuando se puede observar cierta similitud entre los perfiles de los tres grupos (Figura 3), las diferencias entre ellos alcanzaron significancia estadística $[\Lambda$ Wilks $=.75, F(10,246)=3.75, p<.01]$, descartando que exista paralelismo dadas las interacciones o cruces entre líneas. Al igual que con las expectativas de éxito, se puede observar la diferencia de percepciones sobre todo en relación con la improvisación y la lectura, donde estudiantes del grupo formal consideran la improvisación como una actividad difícil $(\mathrm{M}=3.50, \mathrm{DT}=1.14)$ y la lectura como una actividad más sencilla $(\mathrm{M}=2.01$, DT $=1.03)$, en tanto que, en el grupo informal, y en menor medida en el mixto, ocurre lo contrario (ver Figura 3). Nuevamente, excepto por la lectura, las percepciones de dificultad del grupo formal son mayores en el resto de las actividades en comparación con los grupos informal y mixto.

\section{Discusión}

Los resultados de esta investigación, en relación con la motivación y aplicación de estrategias de aprendizaje formal e informal, tanto dentro como fuera de la escuela, sugieren notorias diferencias entre estudiantes en función de sus experiencias formativas y musicales. Estos resultados son un reflejo de las diferencias que han caracterizado la formación de estudiantes dentro de las instituciones de educación superior, en comparación con aquellos formados en contextos de aprendizaje informal, mostrando cómo el alumnado que ha recibido entrenamiento musical formal, particularmente dentro de la tradición clásica, realiza con mucha menor frecuencia actividades de improvisación (Sarath, et al., 2016; Vitale, 2011), tocar de oído (Shifres y Holguín-Tovar, 2015; Varvarigou, 2017), y tocar en grupo, dado que se privilegia el aprendizaje individual bajo el esquema de maestro-estudiante (Hallam, 2018; Holguín-Tovar y Martínez, 2017).

En relación con los intereses profesionales, los integrantes de los grupos informal y mixto muestran una mayor apertura para trabajar en actividades musicales fuera de la escuela, tanto en el campo de la enseñanza como en el de la ejecución. Esto les puede permitir egresar con una mayor experiencia como ejecutantes, además de adquirir una amplia variedad de habilidades y desenvolvimiento en el medio musical, además de mejores bases y una mayor experiencia en el campo docente al terminar sus estudios universitarios, lo que les otorga mejores herramientas para enfrentar al cambiante y muchas veces precario mercado laboral (Bille y Jensen, 2018; Guadarrama, Hualde y López, 2016; Iglesias-Díaz y Villarreal-Jiménez, 2019; Machillot, 2018).

En cuanto al consumo musical, mientras estudiantes del grupo formal reportaron escuchar música clásica con mayor frecuencia, los grupos informal y mixto lo hacen con otros géneros populares como el jazz. Esto era previsible, ya que, como la literatura muestra, existe una fuerte relación entre el aprendizaje informal con la música popular, así como entre el aprendizaje formal y la tradición de la música clásica (Dunbar-Hall y Wemyss, 2000; Green, 2002, 2008; Jaffurs, 2004, 2006; Lebler, 2008; Sarath et al., 2016; Virkkula, 2015; Vitale, 2011; Woody 2007, 2012).

Es fácilmente comprensible el rol de la escuela como formadora de cultura, un lugar donde los estudiantes desarrollan la apreciación por diferentes géneros a lo largo de su carrera universitaria. Sin embargo, lo que sería interesante es observar si con el paso del tiempo esa apertura a nuevos géneros se mantiene o se inhibe, llevando al estudiante a considerar únicamente aquellos géneros estudiados formalmente como los únicos dignos de estudio o de consumo. Esto coincide con lo manifestado con Byo (2018) sobre la preferencia de los ensambles tradicionales o de jazz sobre los de música popular dentro de los ambientes educativos formales. Estos juicios de valor podrían incidir posteriormente en el desempeño laboral del estudiante y sus oportunidades de trabajo.

Mostrar más apertura hacia el consumo de una mayor variedad de géneros musicales, derivaría en una cultura musical más amplia que les puede ofrecer mayores oportunidades y competitividad en el campo laboral, y que, además, les de oportunidades de involucrarse con música que refleje sus intereses (Hallam, Creech y McQueen, 2018). 
Es importante recordar que, de acuerdo con Green (2002), sólo un 3 o 4\% del consumo musical del público general corresponde a música clásica y un porcentaje todavía menor a la música jazz; otros estudios recientes reafirman esta tendencia (AMAI, 2014; Chávez, Urbina y Quevedo, 2019; Ferwerda y Schdell, 2016; Watson, 2020). Resulta pues imperante abogar por una mayor inclusión de otros géneros populares que es el campo laboral donde a final de cuentas se van a desempeñar la mayoría de los egresados.

En cuanto a las limitantes del estudio, no se realizaron mayores cuestionamientos para saber si estas diferencias eran atribuibles a aspectos económicos (necesidad de trabajar), aspectos de autopercepción (que los integrantes del grupo formal se sintieran con menor capacidad para realizar estas actividades), aspectos culturales (que estudiantes del grupo formal no se sintieran capaces de participar en agrupaciones musicales de música popular o que solo quisieran participar en conjuntos clásicos), u oportunidades laborales (que existiera una mayor demanda por aprender música popular en vez de música clásica). Estas situaciones planteadas son sólo algunas de las que se pueden inferir, sin embargo, pudieran no ser las únicas o existir otras de mayor relevancia.

Debido a que solo se recopiló información para realizar un perfil de los participantes y no se hicieron más indagatorias al respecto, se desconoce si tales preferencias son inherentes a ellos en sí mismos y si el ambiente en el que se desarrollaban antes de iniciar su formación era una determinante; o bien, si es atribuible a la formación musical recibida durante sus estudios de licenciatura, que hayan moldeado sus gustos e intereses, por ejemplo, si quienes fueron clasificados dentro del grupo formal ya tenían un gusto por la música clásica o si lo fueron desarrollando durante su formación.

\subsection{Motivación hacia el uso de estrategias de aprendizaje formal e informal}

Los perfiles motivacionales en relación con el uso de estrategias de aprendizaje formal e informal, tanto dentro como fuera de la escuela, mostraron notorias diferencias entre los grupos formal, informal y mixto. Es de destacar que aún en estrategias como la interpretación (tocar la pieza como está escrita), los integrantes del grupo formal muestran menos expectativas de éxito, a pesar de que en su instrucción musical hay un mayor énfasis en ello. Esto puede deberse a las constantes observaciones y correcciones por parte de los profesores en relación con su interpretación que pueden influir negativamente en su sentido de competencia.

El grupo formal muestra además menores expectativas de éxito para todas las estrategias de aprendizaje informal (tocar de oído, improvisar, tocar en grupo). Si bien, estos datos se obtuvieron por medio de autorreportes y no por observación directa, aún con esta limitante, los datos obtenidos tanto en las estadísticas descriptivas como en el análisis de perfiles muestran coincidencia con la literatura revisada, en relación a que en el ambiente formal no se promueve debidamente la creatividad, ni la improvisación (Dolan et al., 2018; Sarath et al., 2016; Vitale, 2011) y, por ende, los músicos no se sienten aptos para realizar estas actividades (Lehmann et al., 2007).

Además del uso de estrategias dentro de la escuela, se recopiló información acerca de la frecuencia del uso de estas estrategias fuera de la escuela, donde los resultados obtenidos fueron similares y siguieron existiendo diferencias significativas entre los grupos informal y mixto en actividades como improvisación y tocar en ensamble en comparación con el grupo formal. Si bien, esta información sirve para complementar los datos y realizar un perfil más completo de quienes participaron en el estudio, los resultados obtenidos nos muestran que el uso de estrategias dentro de la escuela tiene fuerte influencia en lo que el alumnado hace fuera de la misma. Esta influencia en su práctica musical también puede tener efecto en aquellos que luego se dediquen a la docencia. Esto tiene diversas implicaciones, pues existe la posibilidad de que dichas costumbres los lleven a replicar las mismas prácticas con sus estudiantes en un futuro, continuando con las tendencias observadas hasta hoy, razón por lo cual, si se busca tener una mayor apertura con el fin de diversificar las prácticas y estrategias musicales dentro de los ambientes formales, es imperativo empezar a inculcarlas y abogar por su inclusión desde ahora, con el fin de ir modificando la práctica del docente de música y que este cambio vaya permeando a las nuevas generaciones.

Los resultados de los análisis de perfiles sugieren que las percepciones de valor, expectativas de éxito y dificultad de la tarea varían en función del tipo de formación musical. Se puede observar nuevamente cómo los estudiantes del grupo formal valoran más las estrategias relacionadas con la educación musical formal, mientras que los integrantes del grupo informal hacen lo propio con las estrategias informales. Las diferencias más notorias se encuentran en las estrategias de improvisación y lectura. Estos hallazgos encuentran consistencia al contrastarse con lo reportado por el alumnado en cuanto a sus actividades en clase y extra-clase además de que reflejan lo encontrado en la literatura revisada (Green, 2002; Lehmann et al., 2007; Sarath et al., 2016; Vitale, 2011).

En este sentido, se observa cómo en la educación formal se sigue dando preferencia a prácticas como la lectura y la interpretación fiel de la partitura sobre el tocar de oído y la improvisación. Sin embargo, también muestra que existe una cierta incorporación de las estrategias informales dentro de las universidades donde se realizó la investigación, lo cual hace notar que, por lo menos en estas instituciones, ya existe la inquietud de explorar una mayor diversidad de estrategias de aprendizaje musical. Existen aun así limitantes, ya que su aplicación se da principalmente en músicos que estudian géneros de jazz y géneros populares, y en instrumentos comunes a dichos géneros, tales como guitarra eléctrica, bajo eléctrico, batería o saxofón. En contraste, instrumentos que siguen fuertemente ligados a la música clásica, tales como piano y cuerdas siguen las tendencias hacia un tipo de enseñanza tradicional. Por otro lado, se puede encontrar alumnos que participan tanto en géneros clásicos o populares, en casos 
como el piano o bel canto, los cuales reciben algunas veces el apoyo de sus maestros, pero en otras ocasiones se enfrentan a la desaprobación de estos.

Con relación a las percepciones sobre expectativas de éxito y dificultad de la tarea se encontraron diferencias significativas no sólo entre los perfiles de los grupos sino también entre las estrategias de aprendizaje en general. Los grupos informal y mixto presentan mayores expectativas de éxito para todas las estrategias de aprendizaje con excepción de la lectura. Lo mismo sucede en el subconstructo de dificultad de la tarea, aunque de una forma menos marcada, donde el grupo formal en general percibe más dificultad para el uso de todas las estrategias con excepción de la lectura musical.

Lo anterior implica que, por lo general, los integrantes de los grupos informal y mixto tienen una mejor percepción de sus competencias y reportan una mayor confianza para realizar cualquiera de las estrategias de aprendizaje. Esto encuentra un cierto paralelo con lo reportado por Mok (2018), en un estudio en el cual utilizó la estrategia informal de copiar y aprender piezas de oído elegidas por los alumnos para su posterior ejecución, aunque en ese estudio particular, no se encontró una diferencia estadísticamente significativa entre la aplicación de esta estrategia en comparación con las tradicionales, la preferencia por el uso de la estrategia informal por parte de los discentes era casi del doble. Un caso similar ocurre con lo reportado con Varvarigou (2017), dentro de su estudio, se les enseñó a estudiantes de música clásica estrategias para tocar de oído por cinco semanas, $78 \%$ de ellos reportaron al final del curso, sentir una mayor confianza como músicos. Este tipo de resultados indican, que el uso de estrategias informales tiene un impacto positivo en la percepción de las clases, aunado también a una mayor confianza y percepción de las habilidades propias, sin afectar la calidad de la instrucción, factores que sin duda deben ser puestos a consideración.

Es importante reconocer que no se tienen elementos suficientes para aseverar que el tipo de instrucción recibida por parte de los profesores influye en la autopercepción de los alumnos. Sin embargo, como se mencionó en la literatura, es una práctica común en la educación formal que el alumnado presente piezas y constantemente reciba correcciones por parte de sus profesores, lo que puede generar una baja percepción sobre sus propias competencias. Es posible que, al menos inicialmente, los discentes carezcan de una buena percepción de sí mismos y no tengan la suficiente autonomía para la toma de decisiones sobre su propia práctica.

Bajo los argumentos anteriores, se considera necesario revisar dichas prácticas docentes y abogar por alternativas distintas tales como el aprendizaje entre pares, las comunidades de práctica y talleres (Virkkula, 2015; Virkkula y Kunwar, 2016), todo esto con el fin no sólo de formar estudiantes con una mayor apertura hacia las distintas estrategias de aprendizaje, sino también que tengan una mejor concepción de sí mismos, de su práctica musical y con una mayor autonomía en sus decisiones, además de promover un proceso más activo en el quehacer musical, que vaya más allá de meras cuestiones teóricas o mecánicas, desarrollando una mayor conciencia musical y sentido crítico (Motoyama, 2015).

\section{Conclusiones}

Uno de los propósitos para la realización de esta investigación fue aportar más información que permita dimensionar la necesidad de diversificar la inclusión de estrategias de aprendizaje y géneros musicales dentro de la educación formal, con el objetivo de actualizar y complementar las prácticas recurrentes que persisten en la actualidad. Esto puede conducir a la formación de músicos capaces de manejar una más amplia variedad de estrategias de aprendizaje, dotándolos de una mayor autonomía en la toma de decisiones y con una mayor capacidad de dirigir su propio aprendizaje (Costes-Onishi, 2016; Hallam, Creech y McQueen, 2018). Dichos individuos podrían contar, además, con un repertorio y una cultura musical más amplia, abarcando no sólo aquellas corrientes asociadas con la música clásica, sino también géneros de música popular desde finales del siglo XX hasta la actualidad, ampliando su visión y ofreciéndole más herramientas para el mercado laboral actual. Adicionalmente, dichos estudiantes contarían con una mejor autopercepción, derivando en un mayor sentido de competencia hacia diversas actividades musicales más allá de la lectura y memorización de repertorio, tales como la improvisación, el tocar de oído o interpretar música en grupo.

Este cambio de paradigma involucra aplicar la enseñanza de una manera responsiva más que directiva (Green, 2008), y exige una mayor preparación del docente de música, ya que, además de las habilidades esperadas en los currículos actuales, debe contar con la capacidad de integrar una mayor variedad de estrategias de aprendizaje musical, no solo formales sino también informales dentro del currículo universitario. Tal como lo argumentan diversos autores (Byo, 2018; Hallam, Creech y McQueen, 2018; Hewitt, 2018), el docente debe ser capaz de sacar una pieza de oído propuesta por los alumnos, a la par de sugerir cómo ellos mismos pueden mejorar sus habilidades en el instrumento, contar con habilidades tanto para dirección de agrupaciones musicales, composición e improvisación.

Esta propuesta de enseñanza proporciona una mayor apertura y democracia dentro del salón de clases, permitiendo que el alumnado sea más partícipe en su propia formación, lo cual es beneficioso tanto en su autopercepción como en su autonomía. Supone también una revaloración acerca de las estrategias formales, que, si bien siguen siendo muy importantes, deben reconsiderarse con el propósito de que el docente destine espacio y tiempo de su clase a nuevas estrategias con el fin de lograr un nuevo balance en el desarrollo musical de los estudiantes.

El camino por recorrer es largo para lograr cambios significativos en la educación musical en nivel superior. Como en todo tipo de cambios, siempre es posible encontrar resistencia, ya sea por docentes que después de muchos 
años en la docencia no son proclives a cambiar sus prácticas fácilmente, o incluso entre los mismos estudiantes que estén acostumbrados a la enseñanza tradicional que ha caracterizado la educación musical en este nivel educativo. En este sentido, como investigadores en educación, no queda más que continuar en la búsqueda de nuevas alternativas de innovación, reportar los hallazgos fruto de investigaciones rigurosas y proporcionar evidencia empírica que contribuya a fomentar los cambios que la educación musical en nivel superior necesita, para así lograr una mejor formación y profesionalización de los futuros músicos y educadores musicales.

\section{Referencias bibliográficas}

Alessandroni, N. (2013). Pedagogía vocal contemporánea y profesionales prospectivos: Hacia un modelo de diagnóstico en técnica vocal. Boletín de Arte, 13(13), 1-5. http://www.fba.unlp.edu.ar/boa/13/PDF/BOAAlessandroni.pdf

AMAI, Asociación Mexicana de Investigación de Mercado. (2014). México: ¿Qué música nos gusta? https://www.amai.org/blog/ uncategorized/mexico-que-musica-nos-gusta/

Bandura, A. (2005). Guide for constructing self-efficacy scales. En F. Pajares y T. Urdan (Eds.), Self-efficacy beliefs of adolescents (pp. 307-337). Greenwich, Connecticut: Information Age.

Beavers, A. S., Lounsbury, J. W., Richards, J. K., Huck, S. W., Skolits, G. J. y Esquivel, S. L. (2013). Practical considerations for using exploratory factor analysis in educational research. Practical Assessment, Research \& Evaluation, 18, 1-13. https://doi. org/10.7275/qv2q-rk76

Bermúdez, E., y Duque, E. A. (2000). Historia de la música en Santafé y Bogotá 1538-1938. Bogotá: Fundación Música.

Bille, T., y Jensen, S. (2018). Artistic education matters: Survival in the arts occupations. Journal of Cultural Economics, 42 , 23-43. https://doi.org/10.1007/s10824-016-9278-5

Burcet, M. I. (2017). Hacia una epistemología decolonial de la notación musical. Revista Internacional de Educación Musical, 5(1), 129-138. https://doi.org/10.12967\%2FRIEM-2017-5-p129-138

Burnard, P. (2012). Musical creativities in practice. Oxford: Oxford University Press.

Byo, J. L. (2018). "Modern Band" as school music: A case study. International Journal of Music Education, 36(2), $259-269$. https://doi.org/10.1177/0255761417729546

Carrillo, R., y González-Moreno, P. A. (2019). Estrategias de aprendizaje musical formal e informal: Construcción y validación de un instrumento de medición. Revista Internacional de Educación Musical, 7(1), 81-89. https://journals.sagepub.com/doi/ full/10.1177/2307484119878640

Chávez, H. L., Urbina, J. T., y Quevedo, J. A. Á. (2019). Proceso de formación profesional y consumo musical. RECIE Revista Electrónica Científica de Investigación Educativa, 4(2), 1107-1117. https://doi.org/10.33010/recie.v4i2.420

Costes-Onishi, P. (2016). Negotiating the boundaries between the formal and the informal: An experienced teacher's reflective adaptations of informal learning in a keyboard class for at-risk students. British Journal of Music Education, 33(3), 309-326. https://doi.org/10.1017/S0265051716000140

Dolan, D., Jensen, H. J., Mediano, P. A., Molina-Solana, M., Rajpal, H., Rosas, F., y Sloboda, J. A. (2018). The improvisational state of mind: A multidisciplinary study of an improvisatory approach to classical music repertoire performance. Frontiers in Psychology, 9, 1-21. :https://doi.org/10.3389/fpsyg.2018.01341

Dunbar-Hall, P., y Wemyss, K. (2000). The effects of the study of popular music on music education. International Journal of Music Education, 36(1), 23-34. https://doi.org/10.1177/025576140003600104

Eccles, J. S., Adler, T. R, Futterman, R., Goff, S. B., Kaczala, C. M., Meece, J., y Midgley, C. (1983). Expectancies, values and academic behaviors. En J. T. Spence (Ed.), Achievement and achievement motives (pp. 75-146). San Francisco: Freeman.

Eccles, J. S., Midgley, C., Wigfield, A., Buchanan, C. M., Reuman, D., Flanagan, C., y Mac Iver, D. (1993). Development during adolescence: The impact of stage environment fit on young adolescents' experiences in schools and in families. American Psychologist, 48(2), 90-101. https://doi.org/10.1037/0003-066X.48.2.90

Eccles, J. S., y Wigfield, A. (2002). Motivational beliefs, values, and goals. Annual Review of Psychology, 53, 109-132. https:// doi.org/1146/annurev.psych.53.100901.135153

Eccles, J. S., y Wigfield, A. (2020). From expectancy-value theory to situated expectancy-value theory: A developmental, social cognitive, and sociocultural perspective on motivation. Contemporary Educational Psychology (en imprenta). https://doi. org/10.1016/j.cedpsych.2020.101859

Ferwerda, B., y Schedl, M. (2016). Investigating the relationship between diversity in music consumption behavior and cultural dimensions: A cross-country analysis. En Memorias del 24th Conference on User Modeling, Adaptation, and Personalization (UMAP). First Workshop on Surprise, Opposition, and Obstruction in Adaptive and Personalized Systems (SOAP). Halifax, NS, Canadá. http://ceur-ws.org/Vol-1618/SOAP_paper1.pdf

González-Moreno, P. A. (2010). Students' motivation to study music: The Mexican context. Research Studies in Music Education, 32(2), 185-199. https://doi.org/10.1177/1321103X10384211

González-Moreno, P. A. (2012). Student motivation in graduate music programmes: An examination of personal and environmental factors. Music Education Research, 14(1), 79-102. https://doi.org/10.1080/14613808.2012.657168

González-Moreno, P. A. (2015). Music teacher preparation in Mexico: Challenges and opportunities for improvement. En S. Figueiredo, J. Soares y R. Fink-Schambeck (Eds.), The preparation of music teacher: A global perspective, Volume 5 (pp. 99-122). Porto Alegre, Brasil: ANPPOM. 
Green, L. (2002). How popular musicians learn. Aldershot: Ashgate.

Green, L. (2008). Music, informal learning and the school: A new classroom pedagogy. Aldershot: Ashgate.

Guadarrama, R., Hualde, A., y López, S. (2016). Precariedad laboral y trayectorias flexibles en México: Un estudio comparativo de tres ocupaciones. Papers: Revista de Sociología, 101(2), 195-221.

Hallam, S. (2018). Commentary: Instrumental music. En G. E. McPherson y G. F. Welch (Eds.), The Oxford handbook of music education, vol. 1 (pp. 651-657). Oxford: Oxford University Press. https://doi.org/10.1080/14613808.2016.1249358

Hallam, S., Creech, A., y McQueen, H. (2018). Pupils' perceptions of informal learning in school music lessons. Music Education Research, 20(2), 213-230.

Heckel, S. (2017). Soundscapes: Using informal learning pedagogy to create a Canadian strand of musical futures. Canadian Music Educator, 58(2), 12-16.

Hewitt, D. (2018). Constructing informal experiences in the elementary general music classroom. Music Educators Journal, 104(3), 46-53. https://doi.org/10.1177/0027432117745361

Holguín-Tovar, P., y Martínez, I. C. (2017). La didáctica musical entre la primera y la tercera persona: Hacia una perspectiva de segunda persona en la formación de músicos profesionales. Pensamiento, Palabra y Obra, 18, 8-15. https://doi.org/10.17227/ ppo.num18-6279

Iglesias-Díaz, D., y Villarreal-Jiménez, R. (2019). Análisis y propuestas sobre la necesidad de profesionalizar la educación musical en Juárez. Revista del Centro de Investigación y Docencia, Acoyauh Nueva Época, 61, 7-15.

Jaffurs, S. E. (2004). The impact of informal music learning practices in the classroom, or how I learned how to teach from a garage band. International Journal of Music Education, 22(3), 189-200. https://doi.org/10.1177/0255761404047401

Jaffurs, S. E. (2006). The intersection of informal and formal music learning practices. International Journal of Community Music, 4(1), 1-29.

Lebler, D. (2008). Popular music pedagogy: Peer learning in practice. Music Education Research, 10(2), 193-213. https://doi. $\operatorname{org} / 10.1080 / 14613800802079056$

Lehmann, A. C., Sloboda, J. A., y Woody, R. H. (2007). Psychology for musicians: Understanding and acquiring the skills. Oxford: Oxford University Press.

Machillot, D. (2018). The musician's profession, between precarity and redefinition. Sociológica, 33(95), 257-289. http://www. scielo.org.mx/scielo.php?script=sci_arttext\&pid=S0187-01732018000300257\&lng=es\&tlng=en

McCormick, J., y McPherson, G. E. (2007). Expectancy-value motivation in the context of a music performance examination. Musicae Scientiae, 11(2_suppl), 37-52. https://doi.org/10.1177/10298649070110S203

McPherson, G. E. (2009). The role of parents in children's musical development. Psychology of Music, 37(1), 91-110. https://doi. org/10.1177/0305735607086049

McPherson, G. E., y Hendricks, K. S., (2010). Students' motivation to study music: The United States of America. Research Studies in Music Education, 32(2), 201-213. https://doi.org/10.1177/1321103X10384200

McPherson, G. E., y O’Neill, S. A. (2010). Students' motivation to study music as compared to other school subjects: A comparison of eight countries. Research Studies in Music Education, 32(2), 101-137. https://doi.org/10.1177/1321103X10384202

McPherson, G. E., Osborne, M. S., Barrett, M. S., Davidson, J. W., y Faulkner, R. (2015). Motivation to study music in Australian schools: The impact of music learning, gender, and socio-economic status. Research Studies in Music Education, 37(2), 141160. https://doi.org/10.1177/1321103X15600914

Mohd, G., y McPherson, G. E. (2009). Malaysian children's attitudes towards learning music. Music Education Research, 11(2), 193-219. https://doi.org/10.1080/14613800902924474.

Mok, A. O. (2018). Formal or informal — which learning approach do music majors prefer? International Journal of Music Education, 36(3), 380-393. https://doi.org/10.1177/0255761418761258

Motoyama, F. (2015). Informal learning in action: The domains of music teaching and their pedagogic modes. Music Education Research, 19(1), 29-41. https://doi.org/10.1080/14613808.2015.1095866

O’Neill, S. A., y McPherson, G. E. (2002). Motivation. En R. Parncutt y G. McPherson (Eds.), The science and psychology of music performance: Creative strategies for teaching and learning (pp. 31-43). Oxford: Oxford University Press.

Parkes, K. A., y Jones, B. D. (2012). Motivational constructs influencing undergraduate students' choices to become classroom music teachers or music performers. Journal of Research in Music Education, 60(1), 101-123. https://doi. org/10.1177/0022429411435512

Poblete Lagos, C. P. (2019). Formación docente en música en Chile: Una aproximación histórica desde tres universidades. Revista da FAEEBA-Educação e Contemporaneidade, 26(48), 97-109. https://doi.org/10.21879/faeeba2358-0194.2017.v26.n48. p97-109

Puurtinen, M. (2018). Eye on music reading: A methodological review of studies from 1994 to 2017. Journal of Eye Movement Research, 11(2), 1-16. https://doi.org/10.16910/jemr.11.2.2

Sarath, E. W., Myers, D. E. y Campbell, P. S. (2016). Transforming music study from its foundations: A manifesto for progressive change in the undergraduate preparation of music majors. Missoula: College Music Society. http://www.mtosmt.org/issues/ mto.16.22.1/manifesto.pdf

Shifres, F. (2018). Realidad e idealización del dominio de la notación musical. Revista Foro de Educación Musical, Artes y Pedagogía, 4, 13-44.

Shifres, F., y Gonnet D. (2015). Poblematizando la herencia colonial en la educación musical. Epistemus, 3(2), 51-67. 
Shifres, F., y Holguín-Tovar, P. J. (2015). Reconsiderando el desarrollo de las habilidades de audición musical. En F. Shifres y P. J. Holguín-Tovar (Eds.), El desarrollo de las habilidades auditivas de los músicos: Teoría e investigación (pp. 9-19). La Plata, Argentina: GITeV-Grupo de Investigaciones en Técnica Vocal.

Tabachnick, B. G., y Fidell, L. S. (2007). Using multivariate statistics. Boston, MA: Pearson Education.

Uy, R. R. (2018). Student valuations and expectancies for multifarious musical activities. (Doctoral dissertation, Boston University).

Varvarigou, M. (2017). Promoting collaborative playful experimentation through group playing by ear in higher education. Research Studies in Music Education, 39(2), 161-176. https://doi.org/10.1177/1321103X17704000

Virkkula, E. (2015). Informal in formal: The relationship of informal and formal learning in popular and jazz music master workshops in conservatoires. International Journal of Music Education, 34(2), 171-185. https://doi.org/10.1177/0255761415617924

Virkkula, E., y Kunwar, J. B. (2016). Guiding music students during workshop-based on-the-job learning. Journal of Vocational Education \& Training, 69(2), 266-281. https://doi.org/10.1080/13636820.2016.1271995

Vitale, J. L. (2011). Formal and informal music learning: Attitudes and perspectives of secondary school non-music teachers. International Journal of Humanities and Social Science, 1(5), 1-14.

Watson, A. (2020). Distribution of streamed music consumption in the United States in 2019, by genre. https://www.statista.com/ statistics/475667/streamed-music-consumption-genre-usa/

Wigfield, A., y Eccles, J. S. (2000). Expectancy-value theory of achievement motivation. Contemporary Educational Psychology, 25, 68-81. https://doi.org/10.1006/ceps.1999.1015

Wigfield, A., y Eccles, J. S. (2020). 35 years of research on students' subjective task values and motivation: A look back and a look forward. En A. Elliot (Ed.), Advances in motivation science (Vol. 7, pp. 161-198). Amsterdam: Elsevier. https://doi. org/10.1016/bs.adms.2019.05.002

Woody, R. H. (2007). Popular music in school: Remixing the issues. Music Educators Journal, 93(4), 32-37.

Woody, R. H. (2012). Playing by ear: Foundation or frill? Music Educators Journal, 99(2), 82-88. https://doi. org/10.1177/0027432112459199

Wright, R. (2016). Informal learning in general music education. En C. R. Abril C. R., y B. M. Gault (Eds.), Teaching general music: Approaches, issues, and viewpoints (pp. 209-240). Oxford: Oxford University Press. 